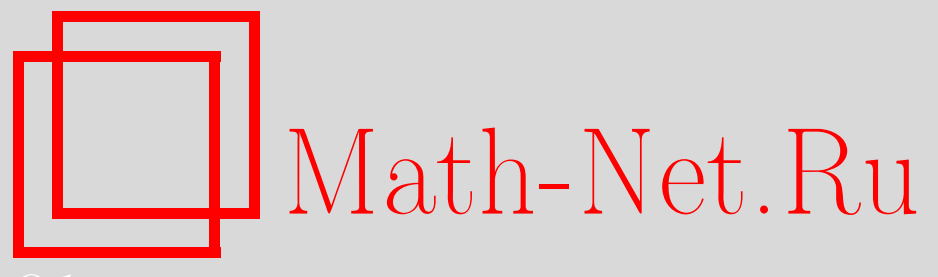

А. В. Шаповалов, Распределения чисел конечных подграфов в случайных неоднородных гиперграфах, Дискрет. матем., 2006, том 18, выпуск 3, 102-114

DOI: https://doi.org/10.4213/dm63

Использование Общероссийского математического портала Math-Net.Ru подразумевает, что вы прочитали и согласны с пользовательским соглашением http: //www . mathnet.ru/rus/agreement

Параметры загрузки:

IP: 3.91 .87 .62

26 апреля 2023 г., $16: 35: 04$ 


\title{
Распределения чисел конечных подграфов в случайных неоднородных гиперграфах
}

\author{
() 2006 г. А. В. Шаповалов
}

\begin{abstract}
Рассматриваются два случайных гиперграфа с $n$ вершинами и $M=M(n)$ ребрами, из которых $M_{i}=M_{i}(n)$ ребер состоят из $i$ неупорядоченных вершин, $i=0,1, \ldots, m$, $M=M_{0}+M_{1}+\ldots+M_{m}$. Выбор вершин для каждого ребра осуществляется случайно, равновероятно из $n$ возможных вершин, для первого случайного гиперграфа по схеме с возвращением, для второго по схеме без возвращения. Исследуются распределения чисел подграфов, изоморфных заданным, при $n \rightarrow \infty, M=M(n)$. Понятия степени и сбалансированности графов распространены на неоднородные гиперграфы. Получена предельная многомерная пуассоновская теорема для чисел строго сбалансированных подграфов, имеющих одинаковую степень. Построена пороговая функция свойства, заключающегося в наличии подграфа, изоморфного произвольному конечному гиперграфу. Для случайных графов такие результаты получены в работах Эрдёша, Реньи, Боллобаша, для случайных однородных гиперграфов (го есть при $M=M_{m}$ ) в работах автора.
\end{abstract}

\section{1. Формулировка основных результатов}

Один из эффективных методов изучения характеристик случайных систем уравнений предполагает использование структурных свойств случайных графов и случайных гиперграфов (см. [4, 5, 9]). В данной работе продолжается начатое в [9] исследование случайных неоднородных гиперграфов, которое в дальнейшем позволит получить широкий спектр асимптотических результатов для случайных линейных и нелинейных систем уравнений.

Под случайным гиперграфом понимается случайный элемент (см. [3]), принимающий значения в некотором множестве гиперграфов в соответствии с некоторой вероятностной мерой. Это множество гиперграфов называется множеством реализаций случайного гиперграфа. Рангом ребра будем называть число инцидентных ему вершин. Число элементов в конечном множестве $Z$ будем, как обычно, обозначать $|Z|$.

Изучаемые случайные гиперграфы имеют $n$ вершин и $M$ ребер. Пусть $m-$ фиксированное натуральное число, $m \geqslant 2$ и

$$
0 \leqslant c_{0}<1, \quad 0 \leqslant c_{i} \leqslant 1, \quad i=1,2, \ldots, m, \quad c_{0}+\ldots+c_{m}=1 .
$$

Множество реализаций случайного гиперграфа $M \widehat{G}_{n, \bar{M}}$ состоит из гиперграфов с множеством вершин $X$ и $M$ ребрами, из которых $M_{i}$ имеют ранг $i, i=0,1, \ldots, m$, $m \geqslant 1, M_{0}+M_{1}+\ldots+M_{m}=M,|X|=n$ (в контексте данного определения полагается 
$\left.\bar{M}=\left(M_{0}, M_{1}, \ldots, M_{m}\right)\right)$. Вершины в ребрах неупорядочены, среди них могут быть совпадающие (применяется выборка вершин по схеме с возвращением), ребра с одинаковыми степенями различимы (или упорядочены, занумерованы). Вероятности всех реализаций одинаковы и равны $\prod_{i=1}^{m}\left(\begin{array}{c}n+i-1 \\ i\end{array}\right)^{-M_{i}}$ (см. [6], с. 220). Такое определение эквивалентно следующей часто используемой форме определения случайного гиперграфа: для каждого $i, i=0,1, \ldots, m$, последовательно случайно и равновероятно выбираются $M_{i}$ ребер с рангом $i$ из всех возможных $\left(\begin{array}{c}n+i-1 \\ i\end{array}\right)$ ребер, имеющих ранг $i$. Ребра, имеющие ранг ноль, все одинаковы.

Случайный гиперграф $M G_{n, \bar{M}}$ определяется аналогично $M \hat{G}_{n, \bar{M}}$ с единственным отличием: выбор $i$ переменных осуществляется не из $\left(\begin{array}{c}n+i-1 \\ i\end{array}\right)$, а из $\left(\begin{array}{l}n \\ i\end{array}\right)$ равновероятных вариантов (выборка без возвращения). Вероятности реализаций случайного гиперграфа $M G_{n, \bar{M}}$ одинаковы и равны $\prod_{i=1}^{m}\left(\begin{array}{c}n \\ i\end{array}\right)^{-M_{i}}$.

Свойства случайных гиперграфов изучаются при $n \rightarrow \infty$, когда $M$ зависит от $n$ некоторым образом, то есть $M=M(n)$, а числа ребер с рангом $i$ следующим образом связаны с константами $c_{i}$ : если $c_{i}=0$, то $M_{i}=0$, если $c_{i}>0$, то $M_{i} \sim c_{i} M, i=1, \ldots, m$. Все асимптотические формулы приводятся при $n \rightarrow \infty$.

Под вероятностью $\mathbf{P}(Q)$ свойства $Q$ реализаций некоторого случайного гиперграфа понимается сумма вероятностей реализаций этого случайного гиперграфа, обладающих свойством $Q$. Эрдёш и Реньи в [13] при изучении случайных графов обнаружили, что многие свойства обладают пороговыми функциями, определяемыми в рассматриваемом случае следующим образом. Если существует функщия $Q(n)$, возрастающая при $n \rightarrow \infty$ и такая, что вероятность свойства $Q$ удовлетворяет условию

$$
\lim _{n \rightarrow \infty} \mathbf{P}(Q)= \begin{cases}1 & \text { при } M(n) / Q(n) \rightarrow \infty, \\ 0 & \text { при } M(n) / Q(n) \rightarrow 0,\end{cases}
$$

то такая функция называется пороговой функцией свойства $Q$, или порогом свойства $Q$ в случайном гиперграфе.

Если свойство гиперграфа, заключающееся в наличии в его реализациях подграфов, изоморфных некоторому гиперграфу (одному из элементов некоторого множества гиперграфов) имеет пороговую функщию, будем называть ее пороговой функцией этого гиперграфа (множества гиперграфов).

Гиперграф называется І-графом, если он содержит $l_{i}$ ребер, имеющих ранг $i$, $i=0,1, \ldots, m$, где $\mathbf{l}=\left(l_{0}, l_{1}, \ldots, l_{m}\right)$ (см. [9]). Говоря об l-графах, будем полагать, что $l_{0}+l_{1}+\ldots+l_{m}=l$. Степенью (средневзвешенной) l-графа $H$ с $k$ вершинами назовем величину $D(H)=\left(\sum_{i=1}^{m} i l_{i}-k\right) / l$. Условие $k \neq \sum_{i=1}^{m} i l_{i}$ выполняется всегда, за исключением неинтересного случая, когда гиперграф состоит из попарно непересекающихся ребер. Максимальной (средневзвешенной) степенью $D_{m}(H)$ гиперграфа $H$ будем называть максимальное значение степеней всех его собственных подграфов. Гиперграф сбалансирован и строго сбалансирован, если его степень, соответственно, не меньше и строго больше его максимальной степени. Будем называть ребро простым, если все инцидентные ему вершины различны, и особым в противном случае. Гиперграф $H$, являющийся l-графом, будем называть допустимым в реализациях случайного гиперграфа, если $c_{i}>0$ для каждого $l_{i}>0, i=1,2, \ldots, m$. Это означает, что в реализациях случайных гиперграфов имеются ребра со всеми рангами, которые имеют ребра гиперграфа $H$.

Теорема 1. Непустой конечный класс конечных гиперграфов $\mathscr{B}_{D}$, допустимых в реализачиях одного из случайных гиперграфов $M G_{n, \bar{M}}, M \widehat{G}_{n, \bar{M}}$ и имеючих максимальную степень $D$, имеет пороговую функцию $n^{D}$. 
Следствие 1. Пусть в условиях теоремы $1 \mathscr{B}_{D}=\left\{H_{1}, \ldots, H_{t}\right\}$ u $\zeta_{n}^{(j)}-$ число допустимых $H_{j}$-графов в реализачиях случайного гиперграфа $M G_{n, \bar{M}}$ или $M \widehat{G}_{n, \bar{M}}, j=1,2, \ldots, t$. Тогда при $M(n) / n^{D} \rightarrow \infty$

$$
\mathbf{P}\left(\zeta_{n}^{(j)} \geqslant \mathbf{E} \zeta_{n}^{(j)} / 2\right)=1+o(1), \quad \mathbf{E} \zeta_{n}^{(j)} \rightarrow \infty
$$

Следствие 2. В случайных гиперграфах $M G_{n, \bar{M}}, M \widehat{G}_{n, \bar{M}}$ nрu $c_{s}+\ldots+c_{m}>0$ nороговая функии гиперграфа, состоящего из двух простых ребер, пересекающихся по $s \geqslant 1$ вериинам, равна $n^{s / 2}$.

Следствие 3. В случайных гиперграфах $M G_{n, \bar{M}}, M \widehat{G}_{n, \bar{M}}$ пороговая функция допустимого гипердерева, имеющего $l \geqslant 1$ ребер, равна $n^{1-1 / l}$.

Следствие 4. $B$ случайных гиперграфах $M G_{n, \bar{M}}, M \widehat{G}_{n, \bar{M}}$ nрu $c_{0}+c_{1}=0$ пороговая функчия связного конечного гиперграфа с единственным чиклом равна $n$.

Следствие 5. В случайном гиперграфе $M \widehat{G}_{n, \bar{M}}$ при $c_{s}>0,1 \leqslant r \leqslant s-1,2 \leqslant s \leqslant m$, особое ребро ранга $s$, состоящее из $s-r$ вершин (как элементов множества $X$ ), имеет пороговую функцию, равную $n^{r}$.

Теорема 2. Пусть $H_{1}, H_{2}, \ldots, H_{t}$ - фиксированные неизоморфные строго сбалансированные гиперграфы, допустимые в реализачиях случайных гиперграфов $M G_{n, \bar{M}} u$ M $\widehat{G}_{n, \bar{M}}$, причем $H_{j}$ имеет $k^{(j)}$ вершин $и l^{(j)}=\sum_{i=1}^{m} l_{i}^{(j)}$ ребер, где $l_{i}^{(j)}-$ число ребер ранга $i, i=1,2, \ldots, m, a^{(j)}-$ порядок группы автоморфизмов, все гиперграфы имеют одинаковую степень $D>0, m \geqslant 1, \zeta_{n}^{(j)}-$ число $H_{j}$-графов в рассматриваемых случайных гиперграфах, $j=1,2, \ldots, t$.

Тогда при $M \sim c n^{D}$, где $c$ - положительная постоянная, для любого натурального $t$ распределение векторной случайной величины $\left(\zeta_{n}^{(1)}, \zeta_{n}^{(2)}, \ldots, \zeta_{n}^{(t)}\right)$ слабо сходится $\kappa$ многомерному распределению Пуассона с независимыми компонентами и вектором средних значений $\left(\lambda_{1}, \lambda_{2}, \ldots, \lambda_{t}\right)$, а для любых чельх неотричательных $s, t_{1}, t_{2}, \ldots, t_{s}$, для которых $t_{1}+t_{2}+\ldots+t_{s}=t$, распределение векторной случайной величины

$$
\left(\sum_{j=1}^{t_{1}} \zeta_{n}^{(j)}, \sum_{j=t_{1}+1}^{t_{1}+t_{2}} \zeta_{n}^{(j)}, \ldots, \sum_{j=t_{1}+t_{2}+\ldots+t_{s-1}+1}^{t} \zeta_{n}^{(j)}\right)
$$

слабо сходится к многомерному распределению Пуассона с независимыми компонентами и вектором средних значений

$$
\left(\sum_{j=1}^{t_{1}} \lambda_{j}, \sum_{j=t_{1}+1}^{t_{1}+t_{2}} \lambda_{j}, \ldots, \sum_{j=t_{1}+t_{2}+\ldots+t_{s-1}+1}^{t} \lambda_{j}\right),
$$

где

$$
\lambda_{j}=\frac{c^{l^{(j)}}}{a^{(j)}} \prod_{i=1}^{m}\left(i ! c_{i}\right)^{l_{i}^{(j)}}, \quad j=1,2, \ldots, t .
$$

Пороговая функция для класса сбалансированных графов получена в [13], для класса сбалансированных однородных гиперграфов анонсирована в [1], для произвольного, вообще говоря, не обязательно конечного графа в $[11,12]$, для произвольного конечного 
однородного гиперграфа в [7]. Ее изменение можно проследить на примере пороговой функции гиперграфа $H$ в $M G_{n, \bar{M}}$. В соответствии с теоремой 1 пороговая функция гиперграфа $H$ в случайном неоднородном гиперграфе $M G_{n, \bar{M}}$ равна $n\left(\sum_{i=1}^{m} i l_{i}-k\right) / l$, где $l_{i}$ - число ребер ранга $i$ в подграфе гиперграфа $H$, имеющем максимальную степень, $i=1,2, \ldots, m ; k$ - число вершин в этом подграфе. Для случайного однородного гиперграфа при $c_{m}=1$ получаем пороговую функцию $n^{m-k / l}$, где $k$ и $l$ - числа вершин и ребер в подграфе гиперграфа $H$, имеющем максимальную степень. При $c_{2}=1$ отсюда получается пороговая функция $n^{2-k / l}$ для случайного графа.

Следствие 1 вносит существенное дополнение к пороговым свойствам конечных подграфов: при $M(n) / n^{D} \rightarrow \infty$ реализации рассматриваемых случайных гиперграфов не только содержат хотя бы один $H_{j}$-граф, но их число стремится к бесконечности со стремящейся к единице вероятностью при $n \rightarrow \infty$.

Первые результаты о пуассоновском распределении чисел строго сбалансированных графов в случайном графе опубликованы в [11, 12]. При развитии другого подхода доказательства результатов такого вида в [10] применена техника нормальной аппроксимации Стейна [14]. Обобщения на случайные однородные гиперграфы получены в [8].

Для доказательства теоремы 1 получаются оценки среднего и дисперсии числа изучаемых подграфов и применяются неравенства Маркова, Чебышёва. Доказательство теоремы 2 проводится методом моментов.

Обзоры работ по случайным графам и гиперграфам можно найти в $[4,5,7,9,12,13]$, определения из теории гиперграфов в [2, 7-9].

\section{2. Доказательства утверждений}

Будем обозначать $V(F)$ и $R(F)$ множества вершин и ребер гиперграфа $F, R_{i}(F)$ множество его ребер, имеющих ранг $i, i=0,1, \ldots, m$.

Введем определение декомпозиции гиперграфа, обобщая соответствующее определение декомпозиции для графа и однородного гиперграфа в смысле Боллобаша (см. [7, $11,12])$. Пусть $H$ - подграф гиперграфа $F$, и $F$ имеет на $k^{*}$ вершин и на $l_{i}^{*}$ ребер ранга $i$ больше, чем $H, i=1,2, \ldots, m$. Дополнительной (средневзвешенной) степенью гиперграфа $F$ над $H$ назовем величину

$$
D(F \mid H)=\left(\sum_{i=1}^{m} i l_{i}^{*}-k^{*}\right) / l^{*} .
$$

Максимальной (средневзвешенной) дополнительной степенью гиперграфа $F$ над $H$ будем называть максимальное значение дополнительных степеней всех подграфов гиперграфа $F$ над $H$ :

$$
m(F \mid H)=\max \left\{D(F \mid H): H \subset F^{\prime} \subset F, V(H) \neq V\left(F^{\prime}\right)\right\}
$$

Определим последовательность подграфов гиперграфа $F: F_{1}$ - подграф, имеющий степень, равную максимальной степени гиперграфа $F$, то есть $D\left(F_{1}\right)=D_{m}(F)$, причем $F_{1}$ не имеет собственных подграфов, обладающих таким свойством. Гиперграф $F_{i+1}$ определяется следующим образом:

$$
V\left(F_{i}\right) \subset V\left(F_{i+1}\right) \neq V(F), \quad V\left(F_{i+1}\right) \neq V\left(F_{i}\right), \quad D\left(F_{i+1} \mid F_{i}\right)=m\left(F \mid F_{i}\right)
$$


Полученную таким образом последовательность $F_{1}, F_{2}, \ldots, F_{t}$, где $F_{t}=F$, будем называть декомпозицией гиперграфа $F$. Из определения следует, что

$$
D\left(F_{1}\right)=D_{m}(F) \geqslant D\left(F_{2} \mid F_{1}\right) \geqslant D\left(F_{3} \mid F_{2}\right) \geqslant \ldots \geqslant D\left(F_{t} \mid F_{t-1}\right)
$$

и то, что не все гиперграфы имеют единственную декомпозицию.

\section{1. Доказательство теоремы 1 и следствий}

Проведем доказательство для $M \widehat{G}_{n, \bar{M}}$. Для второго случайного гиперграфа доказательство полностью повторяется с заменой в формулах величин $\left(\begin{array}{c}n+i-1 \\ i\end{array}\right)$ на величины $\left(\begin{array}{c}n \\ i\end{array}\right)$, $i=1,2, \ldots, m$.

Рассмотрим гиперграф $F$, имеющий подграф $F_{0}$, причем $F$ имеет на $k \geqslant 1$ вершин и $l_{i}$ ребер ранга $i$ больше, чем $F_{0}, i=1,2, \ldots, m$, и $F$ - единственный подграф в $F$ с максимальной дополнительной степенью над $F_{0}$ :

$$
m\left(F \mid F_{0}\right)=D\left(F \mid F_{0}\right)=\left(\sum_{i=1}^{m} i l_{i}-k\right) / l .
$$

Гиперграф $F$ допустим в реализациях случайного гиперграфа $M \widehat{G}_{n, \bar{M}}$, поэтому $l_{i}=0$ при $c_{i}=0, i=0,1, \ldots, m$.

Пусть $V=V_{1} \cup V_{2}, V_{1} \cup V_{2}=\varnothing$ и

$$
\left|V_{2}\right|=n_{2} \sim \theta_{2} n, \quad \theta_{2}>0, \quad M / n_{2}^{\left(\sum_{i=1}^{m} i l_{i}-k\right) / l} \rightarrow \infty
$$

при $n \rightarrow \infty$, и пусть $\widetilde{F}_{0}-$ фиксированный $F_{0}$-гиперграф, множество вершин $V\left(\tilde{F}_{0}\right)$ которого лежит в $V_{1}$.

Докажем, что вероятность того, что имеющие подграф $\widetilde{F}_{0}$ реализации случайного гиперграфа $M \widehat{G}_{n, \bar{M}}$, содержат $F$-граф $H$, имеющий этот подграф $\widetilde{F}_{0}$, стремится к единице, то есть, что

$$
\lim _{n \rightarrow \infty} \mathbf{P}_{M}\left\{M \widehat{G}_{n, \bar{M}} \supset H \supset \tilde{F}_{0}: H \cong F \mid M \widehat{G}_{n, \bar{M}} \supset \tilde{F}_{0}\right\}=1
$$

Из определения $F$ следует, что $k \leqslant \sum_{i=1}^{m} i l_{i}$, причем равенство достигается в том и только том случае, когда $l_{i}$ ребер ранга $i, i=0,1, \ldots, m$, о которых идет речь в определении $F$, изолированы в $F$ (то есть каждое из этих ребер не инцидентно остальными ребрам $F$ ). Учитывая замечание о соотношении величин $c_{i}$ и $l_{i}$, убеждаемся в очевидности утверждения формулы (1) при условии, что $k=\sum_{i=1}^{m} i l_{i}$. Поэтому далее в доказательстве рассматриваем случай $k<\sum_{i=1}^{m} i l_{i}$.

Рассмотрим вероятностное пространство, элементами которого являются все реализации случайного гиперграфа $M \widehat{G}_{n, \bar{M}}$, имеющие на множестве вершин $V\left(\widetilde{F}_{0}\right)$ подграф $\tilde{F}_{0}$. Введем на нем равномерную меру и обозначим соответствующий гиперграф через $M \widehat{G}_{n, \bar{M}}\left(\tilde{F}_{0}\right)$. Несложно убедиться, что $M \hat{G}_{n, \bar{M}}\left(\tilde{F}_{0}\right)$ имеет $\prod_{i=1}^{m}\left(\begin{array}{c}n+i-1 \\ i\end{array}\right)^{M_{i}-l_{i}^{(0)}}$ реализаций, где $l_{i}^{(0)}$ - число ребер ранга $i$ в $F_{0}, i=0,1, \ldots, m$. Для рассматриваемой нами вероятности справедлива оценка

$\mathbf{P}_{M}\left\{M \hat{G}_{n, \bar{M}} \supset H \supset \tilde{F}_{0}: H \cong F \mid M \hat{G}_{n, \bar{M}} \supset \tilde{F}_{0}\right\}=\mathbf{P}_{M}\left\{M \widehat{G}_{n, \bar{M}}\left(\tilde{F}_{0}\right) \supset H \supset \tilde{F}_{0}: H \cong F\right\}$. 
Для доказательства формулы (1) достаточно получить оценку для правой части последнего равенства при $n \rightarrow \infty$. Обозначим через $\zeta_{n}$ число $F$-графов в случайном графе $M \widehat{G}_{n, \bar{M}}\left(\widetilde{F}_{0}\right)$ таких, что $H \supset \tilde{F}_{0}, V(H) \backslash V\left(\tilde{F}_{0}\right) \subset V_{2}$ и существует изоморфизм гиперграфов $H$ и $F$, отображающий $\widetilde{F}_{0}$ в $F_{0}$. Среднее значение случайной величины $\zeta_{n}$ определяется формулой

$$
\mathbf{E} \zeta_{n}=\left(\begin{array}{c}
n_{2} \\
k
\end{array}\right) t \prod_{i=1}^{m}\left(\left(M_{i}-l_{i}^{(0)}\right)_{l_{i}}\left(\begin{array}{c}
n+i-1 \\
i
\end{array}\right)^{M_{i}-l_{i}^{(0)}-l_{i}}\left(\begin{array}{c}
n+i-1 \\
i
\end{array}\right)^{-\left(M_{i}-l_{i}^{(0)}\right)}\right) .
$$

Действительно, число способов выбора недостающих $k$ вершин из множества $V_{2}$ для $H$-графов равно $\left(\begin{array}{c}n_{2} \\ k\end{array}\right) ; t \geqslant 1$ - константа, зависящая только от $F$ и $F_{0}$, равная числу размещений $l_{i}$ ребер ранга $i$ из $\left\{R(F) \backslash R\left(\tilde{F}_{0}\right)\right\}, i=0,1, \ldots, m$, так, чтобы получившиеся $H$-графы были различными (без учета меток ребер); $\left(M_{i}-l_{i}^{(0)}\right) l_{i}-$ число способов выбора номеров для этих $l_{i}$ ребер ранга $i$ из $M_{i}-l_{i}^{(0)}$ номеров; $\left(\begin{array}{c}n+i-1 \\ i\end{array}\right)^{M_{i}-l_{i}^{(0)}-l_{i}}-$ число вариантов выбора оставшихся $M_{i}-l_{i}^{(0)}-l_{i}$ ребер ранга $i ; \prod_{i=1}^{m}\left(\begin{array}{c}n+i-1 \\ i\end{array}\right)^{M_{i}-l_{i}^{(0)}}-$ общее число гиперграфов в $M \widehat{G}_{n, \bar{M}}\left(\widetilde{F}_{0}\right)$.

Убеждаемся, что

$$
\mathrm{E} \zeta_{n} \sim t \frac{n_{2}^{k}}{k !} \prod_{i=1}^{m}\left(\frac{i ! M_{i}}{n^{i}}\right)^{l_{i}}=\theta_{2}^{k} \frac{t}{k !} \prod_{i=1}^{m}\left(i ! c_{i}\right)^{l_{i}}\left(\frac{M}{{ }_{n}\left(\sum_{i=1}^{m} i l_{i}-k\right) / l}\right)^{l} .
$$

Следовательно, в условиях леммы $\mathbf{E} \zeta_{\boldsymbol{n}} \rightarrow \infty$.

Второй факториальный момент случайной величины $\zeta_{n}$ равен математическому ожиданию числа всех упорядоченных пар $\left(H_{1}, H_{2}\right)$ в $F$-графах, удовлетворяющих условиям

$$
H_{i} \supset \widetilde{F}_{0}, \quad\left\{V\left(H_{i}\right) \backslash V\left(\tilde{F}_{0}\right)\right\} \subset V_{2}, \quad i=1,2, \quad H_{1} \neq H_{2}
$$

(при этом ребра учитываются вместе с метками).

Пусть $\Sigma_{1}-$ сумма математических ожиданий таких пар $\left(H_{1}, H_{2}\right)$ в $F$-графах, для которых $V\left(H_{1}\right) \cap V\left(H_{2}\right)=V\left(\widetilde{F}_{0}\right)$. Тогда

$$
\Sigma_{1}=\left(\begin{array}{c}
n_{2} \\
k
\end{array}\right)\left(\begin{array}{c}
n_{2}-k \\
k
\end{array}\right) t^{2} \prod_{i=1}^{m}\left(\left(M_{i}-l_{i}^{(0)}\right)_{2 l_{i}}\left(\begin{array}{c}
n+i-1 \\
i
\end{array}\right)^{-2 l_{i}}\right) \sim\left(\mathbf{E} \zeta_{n}\right)^{2} .
$$

Рассмотрим теперь подграфы $H_{1}, H_{2}$, для которых

$$
\begin{gathered}
\left|V\left(H_{1}\right) \cap V\left(H_{2}\right) \cap V_{2}\right|=s \geqslant 1, \\
0 \leqslant\left|\left\{R_{i}\left(H_{1}\right) \cap R_{i}\left(H_{2}\right)\right\} \backslash R_{i}\left(\tilde{F}_{0}\right)\right|=r_{i}<l,
\end{gathered}
$$

причем под пересечением множеств ребер понимаются ребра, имеющие не только одинаковые множества вершин, но и номера. Среднее значение числа таких пар $\left(H_{1}, H_{2}\right)$ в $F$-графах, для которых $s \geqslant 1$, оценивается сверху величиной

$$
t^{2} \sum_{s=1}^{k}\left(\begin{array}{c}
n_{2} \\
k
\end{array}\right)\left(\begin{array}{c}
n_{2}-k \\
k-s
\end{array}\right)\left(\begin{array}{l}
k \\
s
\end{array}\right) \prod_{i=1}^{m}\left(\left(M_{i}-l_{i}^{(0)}\right)_{2 l_{i}}\left(\begin{array}{c}
n+i-1 \\
i
\end{array}\right)^{-2 l_{i}}\right)=O\left(\frac{1}{n}\left(\mathbf{E} \zeta_{n}\right)^{2}\right)
$$


при $r_{1}+\ldots+r_{m}=r=0$ и величиной

$$
\sum_{1 \leqslant r_{1}+\ldots+r_{m}<l} t^{2} \sum_{1 \leqslant s \leqslant k}\left(\begin{array}{c}
n_{2} \\
k
\end{array}\right)\left(\begin{array}{c}
n_{2}-k \\
k-s
\end{array}\right)\left(\begin{array}{l}
k \\
s
\end{array}\right) \prod_{i=1}^{m}\left(\left(M_{i}-l_{i}^{(0)}\right)_{2 l_{i}-r_{i}}\left(\begin{array}{c}
n+i-1 \\
i
\end{array}\right)^{-\left(2 l_{i}-r_{i}\right)}\right)
$$

при $1 \leqslant r=r_{1}+\ldots+r_{m}<l$. Тогда для

$$
\Sigma_{2}=\mathbf{E} \zeta_{n}^{[2]}-\Sigma_{1}
$$

справедлива оценка

$$
\begin{aligned}
\Sigma_{2} & =O\left(\frac{1}{n}\left(\mathbf{E} \zeta_{n}\right)^{2}\right)+O\left(\left(\frac{M^{\sum_{i=1}^{m} l_{i}}}{n^{\sum_{i=1}^{m} i l_{i}-k}}\right)^{2} \sum_{r_{1}+\ldots+r_{m}=1}^{l-1} \frac{n^{\sum_{i=1}^{m} i r_{i}-s}}{M^{\sum_{i=1}^{m} r_{i}}}\right) \\
& =O\left(\frac{1}{n}\left(\mathbf{E} \zeta_{n}\right)^{2}\right)+O\left(\left(\frac{M}{n\left(\sum_{i=1}^{m} i l_{i}-k\right) / l}\right)^{2 l} \sum_{r_{1}+\ldots+r_{m}=1}^{l-1}\left(\frac{n\left(\sum_{i=1}^{m} i r_{i}-s\right) / r}{M}\right)^{r}\right) .
\end{aligned}
$$

Так как $F$ - единственный подграф в $F$ с максимальной дополнительной степенью над $F_{0}$, заключаем, что

$$
\left(\sum_{i=1}^{m} i r_{i}-s\right) / r<\left(\sum_{i=1}^{m} i l_{i}-k\right) / l
$$

и $M / n\left(\sum_{i=1}^{m} i r_{i}-s\right) / r \rightarrow \infty$ при $n \rightarrow \infty$. Следовательно, $\Sigma_{2}=o\left(\left(\mathbf{E} \zeta_{n}\right)^{2}\right)$, отсюда $\mathbf{D} \zeta_{n} \geq o\left(\left(\mathbf{E} \zeta_{n}\right)^{2}\right)$ и с помощью неравенства Чебышёва получаем, что $\mathbf{P}\left(\zeta_{n}=0\right) \rightarrow 0$, так как

$$
\mathbf{P}\left(\zeta_{n}=0\right) \leqslant \mathbf{P}\left(\left|\zeta_{n}-\mathbf{E} \zeta_{n}\right| \geqslant \frac{\mathbf{E} \zeta_{n}}{2}\right) \leqslant \frac{4 \mathbf{E} \zeta_{n}}{\left(\mathbf{E} \zeta_{n}\right)^{2}}
$$

и $\mathbf{E} \zeta_{n} \rightarrow \infty$ при $n \rightarrow \infty$. Теперь формула (1) следует из формулы (2). Отсюда следует также, что при $M / n^{D} \rightarrow \infty$ справедлива оценка

$$
\mathbf{P}\left(\zeta_{n} \geqslant \frac{\mathbf{E} \zeta_{n}}{2}\right)=1+o(1)
$$

Докажем теорему для класса гиперграфов, состоящего из одного элемента. Общий случай будет следовать из этого частного случая в результате рассмотрения гиперграфа, состоящего из объединения гиперграфов, входящих в класс $\mathscr{B}_{D}$.

Рассмотрим декомпозицию $F_{1} \subset F_{2} \subset \ldots \subset F_{t}=F$ гиперграфа $F$. Не ограничивая общности, можем считать, что $F_{i}$ - единственный подграф в $F_{i}$, имеющий максимальную дополнительную степень над $F_{i-1}, i=2,3, \ldots, t$. Пусть $\eta_{n}-$ число $F_{1}$-графов, являющихся l-графами на $k$ вершинах в случайном гиперграфе. Тогда

$$
\mathbf{E} \eta_{n}=\left(\begin{array}{l}
n \\
k
\end{array}\right) \frac{k !}{a} \prod_{i=1}^{m}\left(\left(M_{i}\right)_{l_{i}}\left(\begin{array}{c}
n+i-1 \\
i
\end{array}\right)^{-l_{i}}\right)
$$

где $a$ - порядок группы автоморфизмов гиперграфа $F_{1}, R_{i}\left(F_{1}\right)=l_{i}, i=1,2, \ldots, m$. Следовательно,

$$
\mathrm{E} \eta_{n} \sim \frac{1}{a} \prod_{i=1}^{m}\left(i ! c_{i}\right)^{l_{i}}\left(\frac{M}{n\left(\sum_{i=1}^{m} i l_{i}-k\right) / l}\right)^{l}
$$


и при $M / n\left(\sum_{i=1}^{m} i l_{i}-k\right) / l \rightarrow 0, n \rightarrow \infty$ с помощью неравенства Маркова убеждаемся в справедливости первой части теоремы:

$$
\varlimsup_{n \rightarrow \infty} \mathbf{P}\left(\eta_{n} \geqslant 1\right) \leqslant \lim _{n \rightarrow \infty} \mathbf{E} \eta_{n}=0
$$

(если нет $F_{1}$-графов, то $F$-графов тем более нет).

Пусть теперь $M / n\left(\sum_{i=1}^{m} i l_{i}-k\right) / l \rightarrow \infty$ при $n \rightarrow \infty$. Разобьем множество вершин $V$ на $t$ непересекающихся частей $V_{1}, V_{2}, \ldots, V_{t}$ таких, что

$$
\bigcup_{i=1}^{t} V_{i}=V, \quad\left|V_{j}\right| \sim n / t, \quad j=1,2, \ldots, t
$$

Из (1) следует, что для $i=2,3, \ldots, t$

$$
\lim _{n \rightarrow \infty} \mathbf{P}\left(M \widehat{G}_{n, \bar{M}}\left[\bigcup_{j=1}^{i} V_{j}\right] \supset F_{i} \mid M \widehat{G}_{n, \bar{M}}\left[\bigcup_{j=1}^{i-1} V_{j}\right] \supset F_{i-1}\right)=1
$$

Здесь $M \widehat{G}_{n, \bar{M}}\left[\bigcup_{j=1}^{i} V_{j}\right] \supset F_{i}$ - событие, заключающееся в том, что на множестве вершин $\bigcup_{j=1}^{i} V_{j}$ случайного гиперграфа $M \widehat{G}_{n, \bar{M}}$ имеется $F_{i}$-граф. Проведя необходимые выкладки (как и при доказательстве формулы (1)), убеждаемся, что

$$
\lim _{n \rightarrow \infty} \mathbf{P}\left(M \hat{G}_{n, \bar{M}}\left[V_{1}\right] \supset F_{1}\right)=1
$$

Из двух последних равенств следует, что

$$
\lim _{n \rightarrow \infty} \mathbf{P}\left(M \widehat{G}_{n, \bar{M}} \supset F_{t}\right)=1,
$$

что завершает доказательство теоремы 1.

Утверждение следствия 1 получено в формуле (3). Для доказательства следствий 2-5 достаточно вычислить максимальные степени приведенных в них гиперграфов и воспользоваться теоремой 1 .

\section{2. Доказательство теоремы 2}

Доказательство первой части утверждения теоремы 2 для случайного гиперграфа $M G_{n, \bar{M}}$ проведем методом моментов в многомерном случае. Для случайного гиперграфа $M \widehat{G}_{n, \bar{M}}$ достаточно в этом доказательстве величины $\left(\begin{array}{c}n \\ i\end{array}\right)$ заменить на $\left(\begin{array}{c}n+i-1 \\ i\end{array}\right), i=1,2, \ldots, m$. Вторая часть утверждения следует из первой и аддитивного свойства распределения Пуассона.

Определим $r$-ю факториальную степень множества $\mathscr{B}$ как множество, элементами которого являются все упорядоченные наборы по $r$ штук элементов $\mathscr{B}$, среди которых нет совпадающих. Обозначать это множество будем $\mathscr{B}^{[r]}$. Пусть $\mathscr{B}_{j}-$ множество всех $H_{j}$-графов в реализациях рассматриваемого случайного гиперграфа, $j=1,2, \ldots, t$. Занумеруем элементы каждого из множеств $\mathscr{B}_{j}$ некоторым образом и обозначим их $S\left(v_{i}^{(j)}\right)$, $i=1,2, \ldots, b_{j}$, где $\left|\mathscr{B}_{j}\right|=b_{j}$. Если $I\left(v_{i}^{(j)}\right)$ - случайная величина, равная индикатору 
события, заключающегося в том, что в случайном гиперграфе имеется $H_{j}$-граф $S\left(v_{i}^{(j)}\right)$, To

$$
\zeta_{n}^{(j)}=\sum_{i=1}^{b_{j}} I\left(v_{i}^{(j)}\right)
$$

Введем обозначение $\bar{v}^{(j)}=\left(v_{1}^{(j)}, \ldots, v_{r_{j}}^{(j)}\right)$. Справедливо равенство

$$
\mathbf{E} \zeta_{1}^{\left[r_{1}\right]} \ldots \zeta_{t}^{\left[r_{t}\right]}=\sum \mathbf{E} I\left(v_{1}^{(1)}\right) \ldots I\left(v_{r_{1}}^{(1)}\right) \ldots I\left(v_{1}^{(t)}\right) \ldots I\left(v_{r_{t}}^{(t)}\right)
$$

где суммирование ведется по всем упорядоченным наборам $\bar{v}=\left(\bar{v}^{(1)}, \ldots, \bar{v}^{(t)}\right)$, для которых соответствующие им наборы подграфов принадлежат декартову произведению факториальных степеней $\mathscr{B}_{1}^{\left[r_{1}\right]} \times \ldots \times \mathscr{B}_{t}^{\left[r_{t}\right]}$.

Разобьем эту сумму на две, $\Sigma_{1}$ и $\Sigma_{2}$. К первой из них отнесем слагаемые, соответствующие наборам индексов $\bar{v}$, которым соответствуют гиперграфы $S\left(v_{1}^{(1)}\right), \ldots, S\left(v_{r_{1}}^{(1)}\right), \ldots$, $S\left(v_{1}^{(t)}\right), \ldots, S\left(v_{r_{t}}^{(t)}\right)$, множества вершин которых не пересекаются, а ко второй - все остальные наборы. Тогда

$$
\begin{aligned}
\Sigma_{1}=\left(\begin{array}{c}
n \\
r_{1} k^{(1)}
\end{array}\right)\left(\begin{array}{c}
n-r_{1} k^{(1)} \\
r_{2} k^{(2)}
\end{array}\right) & \ldots\left(\begin{array}{c}
n-r_{1} k^{(1)}-\ldots-r_{t-1} k^{(t-1)} \\
r_{t} k^{(t)}
\end{array}\right) \\
& \times \prod_{j=1}^{t}\left(\frac{\left(r_{j} k^{(j)}\right) !}{\left(a^{(j)}\right)^{r_{j}}}\right) \prod_{i=1}^{m}\left(\left(M_{i}\right)_{\sum_{j=1}^{t}} r_{j} l_{i}^{(j)}\left(\begin{array}{c}
n \\
i
\end{array}\right)^{-\sum_{j=1}^{t} r_{j} l_{i}^{(j)}}\right) .
\end{aligned}
$$

Действительно, $\left(\begin{array}{c}n \\ r_{1} k^{(1)}\end{array}\right)$ - число способов выбора вершин для $r_{1}$ непересекающихся $H_{1}$-графов, $\left(\begin{array}{c}n-r_{1} k^{(1)} \\ r_{2} k^{(2)}\end{array}\right)$ - число способов выбора вершин для $r_{2}$ непересекающихся $H_{2}$-графов так, чтобы они не имели общих вершин с уже выбранными $r_{1} k^{(1)}$ вершинами, и так далее, наконец, $\left(\begin{array}{c}n-r_{1} k^{(1)}-\ldots-r_{t-1} k^{(t-1)} \\ r_{t} k^{(t)}\end{array}\right)$ - число способов выбора вершин для $r_{t}$ непересекающихся $H_{t}$-графов так, чтобы они не имели общих вершин со всеми выбранными до этого вершинами; величины

$$
\left(r_{j} k^{(j)}\right) ! /\left(\left(a^{(j)}\right)^{r_{j}}\right)=\left(r_{j} k^{(j)}\right) !\left(k^{(j)} !\right)^{r_{j}} /\left(\left(k^{(j)} !\right)^{r_{j}}\left(a^{(j)}\right)^{r_{j}}, \quad j=1,2, \ldots, t,\right.
$$

равны числам различных способов размещения без пересечений $r_{j}$ упорядоченных

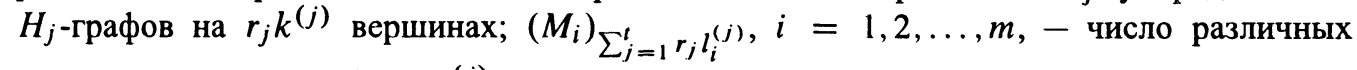
вариантов выбора $\sum_{j=1}^{t} r_{j} l_{i}^{(j)}$ ребер ранга $i$ с учетом их порядка для $H_{j}$-графов, $j=1, \ldots, t$; произведение $\prod_{i=1}^{m}\left(\begin{array}{c}n \\ i\end{array}\right)^{-\sum_{j=1}^{t} r_{j} l_{i}^{(j)}}$ получается как отношение величины $\prod_{i=1}^{m}\left(\begin{array}{c}n \\ i\end{array}\right) M_{i}-\sum_{j=1}^{t} r_{j} l_{i}^{(j)}$, равной числу вариантов выбора оставшихся $M_{i}-\sum_{j=1}^{t} r_{j} l_{i}^{(j)}$ ребер ранга $i, i=1,2, \ldots, m$, в реализациях случайного гиперграфа $M G_{n, \bar{M}}$, к величине $\prod_{i=1}^{m}\left(\begin{array}{c}n \\ i\end{array}\right)^{M_{i}}$, равной общему числу его реализаций. 
Проводя необходимые преобразования, получаем, что

$$
\begin{aligned}
\Sigma_{1} & \sim \prod_{j=1}^{t}\left(\frac{n^{r_{j} k^{(j)}}}{\left(a^{(j)}\right)^{r_{j}}}\right) \prod_{i=1}^{m}\left(\left(i ! c_{i}\right)^{\sum_{j=1}^{t} r_{j} l_{i}^{(j)}} n^{-\sum_{j=1}^{t}\left(r_{j} i l_{i}^{(j)}\right)} M^{\sum_{j=1}^{t} r_{j} l_{i}^{(j)}}\right) \\
& =\prod_{j=1}^{t}\left(\frac{1}{\left(a^{(j)}\right)^{r_{j}}} n^{r_{j}\left(k^{(j)}-\sum_{i=1}^{m} i l_{i}^{(j)}\right)} M^{r_{j} \sum_{i=1}^{m} l_{i}^{(j)}} \prod_{i=1}^{m}\left(\left(i ! c_{i}\right)^{l_{i}^{(j)}}\right)^{r_{j}}\right) \\
& =\prod_{j=1}^{t}\left(\left(\frac{M}{{ }_{n}\left(\sum_{i=1}^{m} i l_{i}^{(j)}-k^{(j)}\right) / l^{(j)}}\right)^{l^{(j)}} \frac{\prod_{i=1}^{m}\left(i ! c_{i}\right)^{l_{i}^{(j)}}}{a^{(j)}}\right)^{r_{j}} .
\end{aligned}
$$

Учитывая, что рассматриваемые гиперграфы $H_{1}, H_{2}, \ldots, H_{t}$ строго сбалансированы, имеют одинаковую степень

$$
D=\left(\sum_{i=1}^{m} i l_{i}^{(j)}-k^{(j)}\right) / l^{(j)}, \quad j=1,2, \ldots, t
$$

и $M \sim c n^{D}$, заключаем, что

$$
\Sigma_{1} \sim \lambda_{1}^{r_{1}} \ldots \lambda_{t}^{r_{t}}
$$

Для завершения доказательства первой части теоремы нам достаточно убедиться в справедливости формулы $\Sigma_{2}=o(1)$.

Пусть $G_{j}$ и $F_{j}$ - некоторые конечные допустимые в реализациях $M G_{n, \bar{M}}$ гиперграфы, $F_{j}$ изоморфен одному из гиперграфов $H_{1}, \ldots, H_{t}$.

Если $0<\left|V\left(F_{j}\right) \cap V\left(G_{j}\right)\right|<\left|V\left(F_{j}\right)\right|$, то в силу строгой сбалансированности $F_{j}$ справедливо неравенство

$$
\frac{\sum_{i=1}^{m} i\left|R_{i}\left(F_{j} \cap G_{j}\right)\right|-\left|V\left(F_{j} \cap G_{j}\right)\right|}{\left|R\left(F_{j} \cap G_{j}\right)\right|}<\frac{\sum_{i=1}^{m} i\left|R_{i}\left(F_{j}\right)\right|-\left|V\left(F_{j}\right)\right|}{\left|R\left(F_{j}\right)\right|}=D,
$$

откуда

$$
\begin{aligned}
\left(\sum_{i=1}^{m} i\left|R_{i}\left(F_{j} \cap G_{j}\right)\right|-\left|V\left(F_{j} \cap G_{j}\right)\right|\right) & \left|R\left(F_{j}\right)\right| \\
& \leqslant\left(\sum_{i=1}^{m} i\left|R_{i}\left(F_{j}\right)\right|-\left|V\left(F_{j}\right)\right|\right)\left|R\left(F_{j} \cap G_{j}\right)\right|-1,
\end{aligned}
$$

и

$$
\sum_{i=1}^{m} i\left|R_{i}\left(F_{j} \cap G_{j}\right)\right|-\left|V\left(F_{j} \cap G_{j}\right)\right| \leqslant D\left|R\left(F_{j} \cap G_{j}\right)\right|-\frac{1}{\left|R\left(F_{j}\right)\right|}
$$

Если $\left|V\left(F_{j}\right) \cap V\left(G_{j}\right)\right|=0$, то

$$
\sum_{i=1}^{m} i\left|R_{i}\left(F_{j} \cap G_{j}\right)\right|-\left|V\left(F_{j} \cap G_{j}\right)\right|=D\left|R\left(F_{j} \cap G_{j}\right)\right|=0 .
$$


В случае $\left|V\left(F_{j}\right) \cap V\left(G_{j}\right)\right|=\left|V\left(F_{j}\right)\right|$ справедливо неравенство

$$
\sum_{i=1}^{m} i\left|R_{i}\left(F_{j} \cap G_{j}\right)\right|-\left|V\left(F_{j} \cap G_{j}\right)\right| \leqslant D\left|R\left(F_{j} \cap G_{j}\right)\right|
$$

Теперь рассмотрим произвольный из $r$-наборов

$$
\left(F_{1}, \ldots, F_{r}\right)=S\left(v_{1}^{(1)}\right), \ldots, S\left(v_{r_{t}}^{(t)}\right),
$$

по которым ведется суммирование в $\Sigma_{2}, r=r_{1}+\ldots+r_{t}$. Введем обозначения

$$
G_{j}=\bigcup_{i=j+1}^{r} F_{i}, \quad j=0,1, \ldots, r-1
$$

При $k \geqslant 1$

$$
\left|R_{i}\left(G_{k-1}\right)\right|=\left|R_{i}\left(F_{k} \cup G_{k}\right)\right|=\left|R_{i}\left(F_{k}\right)\right|-\left|R_{i}\left(F_{k} \cap G_{k}\right)\right|+\left|R_{i}\left(G_{k}\right)\right|,
$$

откуда

$$
\left|R_{i}\left(\bigcup_{k=1}^{r} F_{k}\right)\right|=\left|R_{i}\left(G_{0}\right)\right|=\sum_{k=1}^{r}\left|R_{i}\left(F_{k}\right)\right|-\sum_{k=1}^{r-1}\left|R_{i}\left(F_{k} \cap G_{k}\right)\right|
$$

и

$$
\sum_{i=1}^{m} i\left|R_{i}\left(G_{0}\right)\right|=\sum_{k=1}^{r} \sum_{i=1}^{m} i\left|R_{i}\left(F_{k}\right)\right|-\sum_{k=1}^{r-1} \sum_{i=1}^{m} i\left|R_{i}\left(F_{k} \cap G_{k}\right)\right|
$$

Аналогично получаем формулы

$$
\begin{aligned}
& \left|R\left(G_{0}\right)\right|=\sum_{i=1}^{m}\left|R_{i}\left(G_{0}\right)\right|=\sum_{k=1}^{r} \sum_{i=1}^{m}\left|R_{i}\left(F_{k}\right)\right|-\sum_{k=1}^{r-1} \sum_{i=1}^{m}\left|R_{i}\left(F_{k} \cap G_{k}\right)\right|, \\
& \left|V\left(G_{0}\right)\right|=\sum_{k=1}^{r}\left|V\left(F_{k}\right)\right|-\sum_{k=1}^{r-1}\left|V\left(F_{k} \cap G_{k}\right)\right|
\end{aligned}
$$

Тогда

$$
\begin{aligned}
\sum_{i=1}^{m} i\left|R_{i}\left(G_{0}\right)\right|-\left|V\left(G_{0}\right)\right|=\sum_{k=1}^{r}\left(\sum_{i=1}^{m} i\left|R_{i}\left(F_{k}\right)\right|-\left|V\left(F_{k}\right)\right|\right) \\
\quad-\sum_{k=1}^{r-1}\left(\sum_{i=1}^{m} i\left|R_{i}\left(F_{k} \cap G_{k}\right)\right|-\left|V\left(F_{k} \cap G_{k}\right)\right|\right) .
\end{aligned}
$$

Выше было показано, что для $k=1,2, \ldots, t$

$$
\sum_{i=1}^{m} i\left|R_{i}\left(F_{k} \cap G_{k}\right)\right|-\left|V\left(F_{k} \cap G_{k}\right)\right| \leqslant D\left|R\left(F_{k} \cap G_{k}\right)\right|,
$$


однако в силу определения $\Sigma_{2}$

$$
\left|\bigcup_{i=j+1}^{r} F_{i}\right|=v<k=r_{1} k_{1}+\ldots+r_{t} k_{t}
$$

и существует хотя бы одно число $j$ в множестве $\{1,2, \ldots, r-1\}$, для которого $0<\left|V\left(F_{j}\right) \cap V\left(G_{j}\right)\right|<\left|V\left(F_{j}\right)\right|$, следовательно, для $F_{j}$ и $G_{j}$ справедливо неравенство (4). Из соотношений (4), (5), (6) получаем оценку

$$
\sum_{i=1}^{m} i\left|R_{i}\left(G_{0}\right)\right|-\left|V\left(G_{0}\right)\right| \geqslant D\left(\sum_{k=1}^{r}\left|R\left(F_{k}\right)\right|-\sum_{k=1}^{r-1}\left|R\left(F_{k} \cap G_{k}\right)\right|\right)+\frac{1}{\left|R\left(F_{j}\right)\right|} .
$$

Учитывая формулу

$$
\left|R\left(G_{0}\right)\right| \leqslant \sum_{k=1}^{t} r_{k}\left|R\left(F_{k}\right)\right|
$$

и полученную выше оценку для $\left|R\left(G_{0}\right)\right|$, получаем, что

$$
\begin{aligned}
\frac{\sum_{i=1}^{m} i\left|R_{i}\left(G_{0}\right)\right|-\left|V\left(G_{0}\right)\right|}{\left|R\left(G_{0}\right)\right|} & \geqslant D+\frac{1}{\left|R\left(F_{j}\right)\right|\left|R\left(G_{0}\right)\right|} \\
& \geqslant D+\frac{1}{R_{0} \sum_{k=1}^{t} r_{k}\left|R\left(F_{k}\right)\right|}>D,
\end{aligned}
$$

где $R_{0}$ - максимальное значение $R\left(F_{k}\right)$ для $k=1,2, \ldots, t$.

Среднее значение числа наборов $\left(F_{1}, \ldots, F_{r}\right)$ в сумме $\Sigma_{2}$, удовлетворяющих условиям

$$
\left|V\left(\bigcup_{k=1}^{r} F_{k}\right)\right|=v, \quad\left|R_{i}\left(\bigcup_{k=1}^{r} F_{k}\right)\right|=R_{i}^{(0)}, \quad i=1, \ldots, m,
$$

сверху можно оценить величиной $A\left(n, v, R_{1}^{(0)}, \ldots, R_{m}^{(0)}\right)$, имеющей порядок

$$
\left(\begin{array}{l}
n \\
v
\end{array}\right) \prod_{i=1}^{m}\left(\left(M_{i}\right)_{R_{i}^{(0)}}\left(\begin{array}{l}
n \\
i
\end{array}\right)^{-R_{i}^{(0)}}\right)=O\left(\left(\frac{M}{n\left(\sum_{i=1}^{m} i R_{i}^{(0)}-v\right) \sum_{i=1}^{m} R_{i}^{(0)}}\right)^{\sum_{i=1}^{m} R_{i}^{(0)}}\right) .
$$

Из (7) следует, что

$$
\left(\sum_{i=1}^{m} i R_{i}^{(0)}-v\right) / \sum_{i=1}^{m} R_{i}^{(0)} \geqslant D+\frac{1}{R_{0} \sum_{k=1}^{t} r_{k}\left|R\left(F_{k}\right)\right|}
$$

поэтому

$$
\begin{aligned}
A\left(n, v, R_{1}^{(0)}, \ldots, R_{m}^{(0)}\right) & =O\left(\left(M n^{-\left(D+1 /\left(R_{0} \sum_{k=1}^{t} r_{k}\left|R\left(F_{k}\right)\right|\right)\right)}\right)^{\sum_{i=1}^{m} R_{i}^{(0)}}\right) \\
& =O\left(n^{-\sum_{i=1}^{m} R_{i}^{(0)} /\left(R_{0} \sum_{k=1}^{t} r_{k}\left|R\left(F_{k}\right)\right|\right)}\right)=o(1) .
\end{aligned}
$$


Из этой оценки, учитывая, что набор параметров $v, R_{1}^{(0)}, \ldots, R_{m}^{(0)}$ может принимать конечное число различных значений, убеждаемся, что $\Sigma_{1}=o(1)$. Таким образом, факториальные моменты рассматриваемой векторной случайной величины сходятся к факториальным моментам многомерного распределения Пуассона с независимыми компонентами и указанными в утверждении теоремы средними значениями. Доказательство завершается применением теоремы 21 главы 1 работы [12].

\section{Список литературы}

1. Ванцян А. Г., Эволюция случайных однородных гиперграфов. В сб.: Вероятностные задачи дискретной математики. МИЭМ, Москва, 1987, с. 126-131.

2. Зыков А. А., Гиперграфы. Успехи матем. наук (1974) 29, №6, 89-154.

3. Колчин В. Ф., Случайные отображения. Наука, Москва, 1984.

4. Колчин В. Ф., Системы случайных уравнений. МИЭМ, Москва, 1988.

5. Колчин В. Ф., Случайные графы. Физматлит, Москва, 2004.

6. Сачков В. Н., Введение в комбинаторные методы дискретной математики. Наука, Москва, 2004.

7. Шаповалов А. В., О связности и пороговых функциях подграфов случайных однородных гиперграфов. Дискретная математика (1993) 5, №3, 105-115.

8. Шаповалов А. В., О числе строго сбалансированных подграфов случайных однородных гиперграфов. Дискретная математика (1993) 5, №4, 133-144.

9. Шаповалов А. В., Вероятность совместности случайных систем булевых уравнений. Дискретная математика (1995) 7, №2, 146-159.

10. Barbour A. D., Poisson convergence and random graphs. Math. Proc. Cambridge Phil. Soc. (1982) 92, 349-359.

11. Bollobás B., Threshold functions for small subgraphs. Math. Proc. Cambridge Phil. Soc. (1981) 90, №2, 197-206.

12. Bollobás B., Random graphs. Academic Press, London, 1985.

13. Erdős P., Rényi A., On the evolution of random graphs. Publ. Math. Inst. Hung. Acad. Sci. (1960) 5, 17-61.

14. Stein C., A bound for the error in the normal approximation to the distribution of a sum of dependent random variables. Proc. 6th Berkeley Symp. Math. Stat. Prob. 1970, v.2, 583-602.

Статья поступила 10.06.2005. 